\title{
Économie publique/Public economics
}

économiepublique $06 \mid 2000 / 2$

Efficacité des systèmes éducatifs et de formation.

Vol. 2

\section{Capital humain et croissance : la littérature empirique à un tournant?}

Marc Gurgand

\section{OpenEdition}

\section{Journals}

Édition électronique

URL : http://journals.openedition.org/economiepublique/1636

DOI : 10.4000/economiepublique.1636

ISSN : 1778-7440

Éditeur

IDEP - Institut d'économie publique

Édition imprimée

Date de publication : 15 juillet 2000

ISBN : 2-8041-3384-2

ISSN : 1373-8496

\section{Référence électronique}

Marc Gurgand, "Capital humain et croissance : la littérature empirique à un tournant ? 》, Économie publique/Public economics [En ligne], 06 | 2000/2, mis en ligne le 07 décembre 2005, consulté le 21 décembre 2020. URL : http://journals.openedition.org/economiepublique/1636 ; DOI : https://doi.org/ 10.4000/economiepublique.1636 


\section{économiepublique}

Revue de l'Institut d'Économie Publique

Deux numéros par an

$n^{\mathbf{0}} \mathbf{6}_{-2000 / 2}$

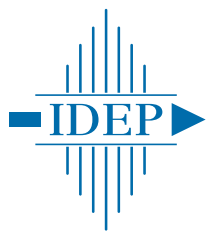


(C) De Boeck \& Larcier s.a., 2002

Editions De Boeck Université

Rue des Minimes 39, B-1000 Bruxelles

Tous droits réservés pour tous pays.

Il est interdit, sauf accord préalable et écrit de l'éditeur,de reproduire (notamment par photocopie) partiellement ou totalement le présent ouvrage, de le stocker dans une banque de données ou de le communiquer au public, sous quelque forme et de quelque manière que ce soit.

Imprimé en Belgique

Dépôt légal 2002/0074/101

ISSN 1373-8496

ISBN 2-8041-3384-2 
économiepublique sur internet : www.economie-publique.fr

(C) Institut d'économie publique - IDEP

Centre de la Vieille-Charité

2, rue de la Charité - F-13002 Marseille

Tous droits réservés pour tous pays.

Il est interdit, sauf accord préalable et écrit de l'éditeur, de reproduire (notamment par photocopie) partiellement ou totalement le présent ouvrage, de le stocker dans une banque de données ou de le communiquer au public, sous quelque forme et de quelque manière que ce soit.

La revue economiepublique bénéficie du soutien du Conseil régional Provence-AlpesCôte d'Azur

ISSN 1373-8496 


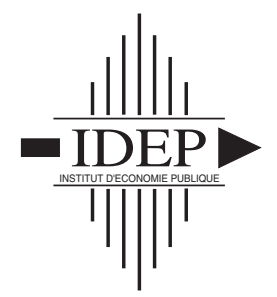

\section{Capital humain et croissance : la littérature empirique à un tournant ?}

Marc Gurgand*

Centre d'études de l'emploi et Crest (Insee)**

\section{Introduction}

Il semblait acquis depuis les années 1960, mais sur la base d'exercices essentiellement comptables, que la croissance du capital humain était une composante importante de la croissance économique et que celui-ci avait par conséquent une place légitime dans la fonction de production agrégée. Ce résultat, pourtant conforme à l'intuition, semble remis en cause par un ensemble de contributions empiriques récentes. L'examen du rôle du capital humain dans la croissance est en effet revenu à l'ordre du jour à l'occasion du débat sur la convergence des économies. Le modèle de Solow, dans lequel le moteur de la croissance est le progrès technique, commun à toutes les économies, prédit une convergence internationale des revenus par tête. Or cette prédiction est peu conforme aux faits, ce qui légitimerait les modèles, en quelque sorte concurrents, de croissance endogène. En 1992, Mankiw, Romer et Weil montrent pourtant qu'il est possible d'amender le modèle classique de Solow en introduisant le capital humain aux cotés du capital physique: cette version du modèle semble alors compatible avec les données.

Par cette voie, le capital humain est donc redevenu un élément clef et un enjeu important de la littérature sur la croissance économique. Des efforts ont été développés pour constituer des bases de données

* L'auteur tient à remercier Jules Nyssen, Pierre Granier et un rapporteur anonyme pour leurs commentaires sur une version antérieure de ce texte.

** Centre d'études de l'emploi et Crest. Adresse : 29, promenade Michel Simon, 93166 Noisy-leGrand cedex; gurgand@cee.enpc.fr. 
internationales de stock de capital humain. Surtout, un ensemble d'auteurs ont estimé de nombreuses spécifications avec diverses méthodes, pour tester la robustesse des résultats de Mankiw, Romer et Weil. On cherchera ici à démêler cette littérature et à clarifier autant que possible le débat en distinguant les classes de modèles et les types de méthodes économétriques utilisés par les uns et les autres et en comparant et discutant les hypothèses retenues dans les différentes approches. Nous montrerons que cette littérature, qui se donne pour objectif d'estimer un effet de l'éducation sur la croissance commun à tous les pays, est incapable de démontrer empiriquement l'existence d'une relation positive. Quelques auteurs suggèrent toutefois que les rendements de l'éducation sont probablement très hétérogènes; ils sont peu nombreux, mais leurs résultats sont prometteurs. Ils alimentent l'idée selon laquelle la productivité du capital humain est, plus que celle d'un autre facteur, sensible à l'environnement économique. Il y a là non seulement un enjeu théorique - puisqu'il est important de pouvoir démontrer que le capital humain peut avoir des effets sur la croissance - mais aussi un enjeu considérable de politique économique : l'investissement en capital humain ne serait vraiment rentable que dans certaines circonstances.

Nous nous limitons ici à la présentation des recherches les plus significatives : il ne s'agit pas de proposer une bibliographie complète mais de mettre en lumière les principaux arguments. Pour la clarté de la présentation, il nous arrivera également de fragmenter les contributions d'un même article lorsque celui-ci procède par étapes en utilisant tour à tour différentes spécifications. Enfin, il est entendu que le terme de capital humain recouvre en général d'autre notions que la seule scolarisation (l'expérience, la formation continue ou encore la santé). Dans la littérature macroéconomique, cependant, il désigne principalement l'éducation (plus rarement la santé), aussi retiendrons-nous ce terme de manière conventionnelle.

Nous rappelons d'abord la structure du modèle néoclassique de croissance (section 2) avant de présenter les différentes spécifications qui ont été utilisées dans la littérature et les résultats empiriques qu'elles ont permis d'obtenir (section 3). Nous discutons ensuite les problèmes strictement économétriques soulevés par ces estimations (section 4) puis nous présentons les quelques travaux qui permettent peut-être de sortir de l'impasse où se trouve la littérature (section5) avant de conclure (section 6). 


\section{Fonction de production agrégée, équilibre, convergence}

Les principales contributions récentes à la littérature empirique sur le rôle du capital humain dans la croissance économique ont pour point de départ une version élargie du modèle de croissance néoclassique de Solow (1956) et Swan (1956) proposée par Mankiw, Romer \& Weil (1992) et c'est pourquoi il est utile d'en rappeler brièvement la structure. Les principes de ce modèle sont bien connus ${ }^{1}:$ il décrit l'équilibre stationnaire d'une économie concurrentielle fermée, lorsque la fonction de production agrégée est à rendements d'échelle constants, lorsque la productivité marginale de chacun des facteurs accumulables est décroissante et lorsque les taux d'épargne sont constants et exogènes. On montre alors que, en l'absence de progrès technique, le taux d'accumulation des facteurs (par tête) serait nul à l'équilibre et l'économie croîtrait uniquement à proportion de l'augmentation de la population. La croissance observée du revenu par tête doit alors s'expliquer par le progrès technique : il est la seule source de croissance de l'économie à l'équilibre.

Soit $Y$ le revenu agrégé, $K$ le stock de capital physique, $L$ la quantité de travail (assimilée ou supposée proportionnelle à la population) et $H$ le stock de capital humain. On suppose que ces quantités sont reliées par une fonction de production à rendements constants qui décrit la technique de production,

$$
Y=F(K, H, A L)
$$

où $A$ est une mesure du progrès technique neutre au sens de Harrod ${ }^{2}$.

L'hypothèse de rendements constants permet d'écrire cette relation en exprimant les grandeurs "par unité efficace de travail", soit

$$
y=f(k, h)
$$

où $y=Y / A L, k=K / A L$ et $h=H / A L$. Les estimations paramétriques que nous allons présenter et discuter ensuite s'appuient toutes sur une spécification Cobb-Douglas de cette fonction, aussi allons-nous l'adopter dès maintenant (bien que ce ne soit pas nécessaire pour dériver les principales propriétés du modèle). La fonction de production s'écrit par conséquent

$$
y=k^{\alpha} h^{\beta}
$$

1 On peut se reporter aux présentations synthétiques de Barro \& Sala-i-Martin (1995) et Aghion \& Howitt (1998). Notre objectif étant de présenter la littérature empirique, nous nous limitons aux types de modèles qui sont estimés, même si ceux-ci peuvent sembler en retrait par rapport aux développements de la littérature sur la croissance.

2 Les autres spécifications classiques du progrès technique, neutre au sens de Hicks ou de Solow ne permettent pas de générer un équilibre stationnaire dans une économie concurrentielle, voir Barro \& Sala-i-Martin (1995). 
avec $\alpha+\beta<1$ parce que la technique de production est à rendements décroissants dans les seuls facteurs $K$ et $H$. Si une proportion $s_{k}$ du produit est investie dans le capital physique et si $\delta$ mesure le taux de dépréciation du capital, on peut écrire l'équation dynamique suivante

$$
\dot{k}=s_{k} y-(n+g+\delta) k
$$

où $\dot{k}=d k / d t$ décrit la variation de $k$ au cours du temps, $n$ est le taux de croissance de la population et $g=(1 / A) d A / d t$ est le taux de croissance du progrès technique ${ }^{3}$. Le capital par unité de travail efficace croît donc avec le taux d'investissement et le niveau de produit mais sa croissance est réduite par la croissance de la population, par le progrès technique et par sa propre dépréciation. Mankiw, Romer \& Weil (1992) proposent d'étendre cette relation au capital humain, en le traitant de façon parfaitement homogène au capital physique, soit

$$
\dot{h}=s_{h} y-(n+g+\delta) h
$$

où $s_{h}$ est la part du produit investie dans le capital humain et $\delta$ est le taux de dépréciation du capital humain, identique à celui du capital physique. La dynamique décrite par le système d'équations différentielles (2.1) et (2.2) fait converger l'économie vers un équilibre stationnaire décrit par les niveaux d'équilibre

$$
\left\{\begin{array}{l}
k^{*}={\frac{s_{k}^{1-\beta} s_{h}^{\beta}}{n+g+\delta}}^{1 /(1-\alpha-\beta)} \\
h^{*}={\frac{s_{k}^{1-\alpha} s_{h}^{\alpha}}{n+g+\delta}}^{1 /(1-\alpha-\beta)}
\end{array}\right.
$$

En replaçant ces valeurs d'équilibre dans la fonction de production et en prenant les logarithmes, il vient la relation d'équilibre de long terme

$$
\log \left(\frac{Y}{L}\right)=\log A+\alpha \log k^{*}+\beta \log h^{*}
$$

dont la forme réduite s'écrit (en exprimant $k^{*}$ et $h^{*}$ en fonction de leurs déterminants exogènes)

$$
\log \left(\frac{Y}{L}\right)=\log A+\alpha^{\prime} \log s_{k}+\beta^{\prime} \log s_{h}-\gamma \log (n+g+\delta)
$$

où $\alpha^{\prime}, \beta^{\prime}$ et $\gamma$ sont des paramètres positifs, fonctions de $\alpha$ et $\beta$ dont la structure particulière n'a pas d'importance pour ce qui suit.

3 Il suffit d'écrire la relation en niveau, $\dot{K}=s_{k} Y-\delta K$, et de réarranger. 
Cette relation ne peut être utilisée pour tester empiriquement le modèle que si l'on considère que les économies ont atteint leur équilibre stationnaire. Dans le cas contraire, il est encore possible d'écrire une approximation du taux de croissance de l'économie à proximité de l'équilibre stationnaire comme

$$
\frac{\dot{y}}{y}=\lambda\left(\log y^{*}-\log y\right)
$$

où $y^{*}$ est la valeur d'équilibre de $y$ et $\lambda=(n+g+\delta)(1-\alpha-\beta)$. De cette équation différentielle, on peut déduire la relation dynamique

$$
\log y_{t}=\left(1-e^{-\lambda t}\right) \log y^{*}+e^{-\lambda t} \log y_{o}
$$

où $t$ mesure le temps et $y_{o}$ est le revenu par unité de travail efficace à la date $t=0$. Cette relation peut s'écrire en forme structurelle et en forme réduite comme

$$
\begin{aligned}
\log \left(\frac{Y_{t}}{L_{t}}\right)= & \log A_{t} \\
= & \left(1-e^{-\lambda t}\right)\left(\alpha \log k^{*}+\beta \log h^{*}\right)+e^{-\lambda t} \log y_{o} \\
& +\left(1-e^{-\lambda t}\right)\left(\alpha^{\prime} \log s_{k}+\beta^{\prime} \log s_{h}-\gamma \log (n+g+\delta)\right) \\
& +e^{-\lambda t} \log y_{o}
\end{aligned}
$$

Ainsi, il suffit de conditionner sur un niveau de revenu initial pour avoir, à proximité de l'équilibre stationnaire, la même relation (à un changement d'échelle des coefficients près) qu'à l'équilibre stationnaire lui-même. L'enjeu de cette dernière spécification est à rechercher dans le débat sur la convergence et la vitesse de convergence des économies : il est en effet essentiel, pour analyser empiriquement le processus de convergence, de décrire les économies hors de leur équilibre de long terme. Pour ce qui nous occupe, cependant, c'est-à-dire la mesure du rôle du capital humain dans la croissance, cette spécification a principalement l'intérêt d'être plus réaliste que les équations (2.3) ou (2.4) parce qu'elle n'impose pas que l'ensemble des économies soient sur leur sentier de croissance équilibrée.

\section{Les estimations du modèle de croissance néoclassique}

La question qui nous intéresse ici est la suivante : dans quelle mesure la littérature empirique qui s'appuie sur les types de modèles présentés dans la section 2 fournit-elle une estimation crédible du rôle du capital humain dans la croissance, c'est-à-dire du paramètre $\beta$ ou d'un de ses dérivés? Il est d'abord utile de récapituler les différentes stratégies 
d'estimation susceptibles d'être appliquées aux différentes spécifications. On indicera par la suite les pays par $i$ et on posera $A_{i t}=A_{o} e^{g t}$ avec $\log A_{o}=a+\varepsilon$, où $\varepsilon$ est un terme résiduel aléatoire et $a$ une constante. Le progrès technique exogène $g$ est donc supposé commun à tous les pays. Par ailleurs, les notations, $\widetilde{y}, \widetilde{k}$ et $\widetilde{h}$ désigneront dorénavant les quantités par tête qui sont mesurables (alors que les quantités par unité efficace de travail ne le sont pas), soit $Y / L$, etc ${ }^{4}$.

La procédure la plus directe consiste à estimer la fonction de production agrégée,

$$
\log \widetilde{y}_{i t}=\alpha \log \widetilde{k}_{i t}+\beta \log \widetilde{h}_{i t}+a+g t+\varepsilon_{i t}
$$

Comme toute relation structurelle cette équation pose des problèmes d'identification puisqu'il est douteux que les stocks de capital physiques et humains accumulés à chaque instant soient indépendants des chocs sur la productivité contenus dans $\varepsilon_{i t}{ }^{5}$. Cette approche requiert donc en principe des méthodes instrumentales qui peuvent être mises en œuvre à condition de disposer de l'information nécessaire dans les données. En revanche, cette fonction a l'avantage de décrire une relation technique et d'être très économe en hypothèses sur l'état de l'économie. Elle est le plus souvent exprimée en taux de croissance, soit,

$$
\log \widetilde{y}_{i t}-\log \widetilde{y}_{i o}=\alpha\left(\log \widetilde{k}_{i t}-\log \widetilde{k}_{i o}\right)+\beta\left(\log \widetilde{h}_{i t}-\log \widetilde{h}_{i o}\right)+g t+\varepsilon_{i t}-\varepsilon_{i o}
$$

À l'opposé, la forme réduite de l'équilibre stationnaire permet d'écrire le revenu en fonction de variables supposées exogènes, les taux d'épargne, mais à condition de faire une hypothèse très forte sur l'état de l'économie:

$$
\log \widetilde{y}_{i t}=\alpha^{\prime} \log s_{k i}+\beta^{\prime} \log s_{h i}-\gamma \log \left(n_{i t}+g+\delta\right)+a+g t+\varepsilon_{i t}
$$

Dans une estimation en coupe transversale, $g$ ne peut pas être identifié ( $g t$ est commun à toutes les observations et entre dans la constante) tandis que $\delta$ est en général mal connu, aussi est-il nécessaire de faire des hypothèses sur la valeur de $(g+\delta)$.

Si on suppose enfin que les économies sont peu éloignées de l'équilibre stationnaire, on a la relation dite "de convergence"

$$
\log \widetilde{y}_{i t}=\alpha^{\prime \prime} \log s_{k i}+\beta^{\prime \prime} \log s_{h i}-\gamma^{\prime} \log \left(n_{i t}+g+\delta\right)+\theta \log \widetilde{y}_{i o}+a+g t+\varepsilon_{i t}
$$

4 Pour simplifier l'écriture, et faciliter la comparaison entre les différentes spécifications, nous ne tiendrons pas compte des renormalisations du type $A_{o}$ en $A_{o}^{1-\alpha-\beta}$ et $g$ en $(1-\alpha-\beta) g$ qui sont impliquées par ce changement de notation et qui modifient les valeurs d'une constante et d'un paramètre sur lesquels nous ne concentrons pas notre attention.

5 Dans un article classique Zellner, Kmenta \& Drèze (1966) montrent cependant que les moindres carrés ordinaires constituent une méthode d'estimation légitime lorsque le résidu $\varepsilon$ est un aléa que les producteurs ne connaissent pas au moment où ils effectuent leurs choix de production. 
On trouve également dans la littérature une spécification intermédiaire, où $s_{h i}$ est remplacé par $h_{i}^{*}$ :

$$
\log \widetilde{y}_{i t}=\alpha^{\prime \prime} \log s_{k i}+\beta^{\prime \prime \prime} \log h_{i}^{*}-\gamma^{\prime} \log \left(n_{i t}+g+\delta\right)+\theta \log \widetilde{y}_{i o}+a+g t+\varepsilon_{i t}
$$

dont nous discuterons bientôt les justifications et les limites. Dans l'une et l'autre de ces deux équations on peut mettre à gauche le taux de croissance du revenu par tête (au lieu de son niveau), ce qui conduit simplement à remplacer $\theta$ par $(\theta-1)$ dans le terme de droite. Trois spécifications peuvent donc être utilisées pour mesurer le rôle du capital humain sur la croissance, la fonction de production, le modèle d'équilibre et le modèle de convergence; en pratique la première et la dernière des trois sont surtout utilisées dans la littérature empirique.

L'estimation des modèles (3.3) et (3.4) a été réalisée par Mankiw, Romer \& Weil (1992). Ils prennent comme mesure du taux d'investissment en capital humain la proportion de la population active potentielle inscrite dans l'enseignement secondaire, en pourcentage sur la période 1965-1985 et expliquent soit le revenu par tête en 1985, soit son taux de croissance entre les deux dates. Barro (1991) avait proposé une estimation du modèle (3.4) en utilisant les taux de scolarisation primaire et secondaire en 1960 (et en incluant un grand nombre de variables de contrôle, dépenses gouvernementales, stabilité politique, etc., en plus de celles requises par le modèle). Ces estimations sont présentées dans le Tableau 1 et indiquent sans ambiguité une relation positive entre la production agrégée (ou la croissance) et ces mesures du capital humain.

Il est peut-être légitime de considérer que les travaux empiriques sur données macroéconomiques sont peu crédibles, l'article de Levine et Renelt (1992) ayant jeté un doute général sur l'analyse empirique de la croissance en coupe transversale. Observant qu'une corrélation positive avec la croissance économique a été obtenue dans la littérature pour plus de 50 variables, ces auteurs ont montré qu'aucune corrélation - sauf pour le taux d'investissement, le PIB initial et le taux de scolarisation secondaire - n'est cependant robuste à des changements de spécification. Sala-i-Martin (1997) nuance toutefois cette conclusion en utilisant un critère de robustesse moins fort que celui de ces deux auteurs. En outre, nous nous concentrons ici sur des estimations limitées aux variables qui semblent précisément résister à ces tests de robustesse.

En faisant abstraction des problèmes strictement économétriques qui seront discutés plus loin, ces résultats ont cependant deux défauts principaux. D'une part, ils font une hypothèse forte sur l'accumulation du capital humain, dont les mécanismes sont probablement beaucoup plus complexes que ceux du capital physique (Cohen, 1996, Dessus, 1998). D'autre part, ils font des hypothèses également contestables sur la proximité de toutes les économies à leur équilibre stationnaire et sur le taux de progrès technique supposé identique pour toutes. Il 
y a en outre une pure incohérence dans l'estimation des modèles de convergence puisque $\theta=e^{-\lambda t}$ est estimé comme un paramètre commun à tous les pays alors que $\lambda$ est une fonction de $n$ dont la variation est par ailleurs prise en compte pour estimer le paramètre $\gamma$. Autrement dit, l'hypothèse selon laquelle tous les pays convergent vers leur équilibre à la même vitesse est non seulement une hypothèse forte mais constitue également une incohérence interne, puisqu'elle est contredite par le modèle lui-même. Pour éviter ce deuxième ensemble de problèmes, la solution consiste à estimer directement une fonction de production, soit les équations (3.1) ou (3.2). Pour celà, il faut disposer de données internationales sur les stocks de capital humain. La première difficulté, quant à elle, peut être contournée en estimant l'équation (3.5), laquelle ne fait pas d'hypothèse sur le processus qui relie le stock de capital humain d'équilibre et les caractéristiques exogènes de l'économie. À nouveau, il faut disposer de données de stock.

En réponse à ces problèmes, qui fixent les limites des résultats publiés au début des années 1990, plusieurs auteurs se sont efforcés de constituer des données de stock de capital humain permettant des comparaisons internationales sur longue période (Kyriacou, 1991, Lau, Jamison \& Louat, 1991, Lau, Bhalla \& Louat, 1991, et surtout Barro \& Lee, 1993 et Nehru, Swanson \& Dubey, 1995). Ces stocks sont exprimés en nombre d'années d'éducation de la population adulte et ils sont calculés à partir de l'empilement des flux (décrits par les taux de scolarisation), en utilisant des tables de mortalité pour tenir compte des flux de disparition du capital humain. Barro \& Lee (1993) s'appuient sur les stocks observés à certaines dates à l'occasion de recensements tandis que Nehru, Swanson \& Dubey (1995) n'utilisent que des flux et remontent à 1902 ou 1930 (pour la plupart des pays) pour estimer le stock de départ en 1960. Les données des premiers sont quinquennales sur la période 1960-1985, celles des seconds sont annuelles sur la période 1960-1987. Nous reviendrons plus loin sur la comparabilité et la fiabilité des différentes séries ainsi obtenues.

La publication de ces données a été suivie d'une deuxième génération d'estimations sur des formes plus structurelles, donc plus robustes du point de vue des hypothèses économiques. Ainsi, le modèle (3.5) a été estimé par Islam (1995) avec les données de Barro \& Lee (1993). Cet auteur propose de mesurer la valeur d'équilibre $h^{*}$ par la valeur observée à la date $t$. Il faut donc admettre que ce stock de capital humain constitue une bonne approximation du stock d'équilibre (ce qui est acceptable si on considère par ailleurs que les économies sont proches de leur équilibre stationnaire); il faut en outre s'assurer que cette quantité n'est pas endogène ${ }^{6}$. En revanche, on ne peut pas justifier cette spécification en considérant naïvement que le stock de capital humain en $t$ doit bien déterminer la production à cette date, car

6 Dessus (1998) qui estime des spécifications proches de celles d'Islam (1995) présente des tests qui ne permettent pas de rejeter l'exogénéité. 
il s'agit alors d'estimer une fonction de production et la présence du terme retardé $\widetilde{y}_{i o}$ resterait à justifier. Le résultat de l'estimation d'Islam économétriquement comparable à celle de Mankiw, Romer \& Weil (1992) se trouve dans le tableau 1. Le coefficient reste positif et significatif (quoique de façon moins nette mais sur un échantillon légèrement plus petit, 79 observations au lieu de 98); il devient non-significatif pour le sous-ensemble des pays à revenus intermédiaires alors qu'il restait significatif chez Mankiw, Romer \& Weil. Ces résultats (qui ne constituent pas la contribution d'Islam, laquelle sera présentée plus loin) indiquent que le passage de la forme réduite à une forme plus structurelle change assez peu de choses tant que l'on reste dans une logique de proximité de l'équilibre stationnaire.

Des modifications plus spectaculaires interviennent lorsque les auteurs utilisent les données de stock pour estimer directement des fonctions de production, de manière à limiter les hypothèses sur l'état d'équilibre des économies. C'est le cas de Benhabib \& Spiegel (1994) qui utilisent les données de Kyriacou (1991) ${ }^{7}$. Ils estiment la spécification (3.2) sur la période 1965-1985, à ceci près qu'ils n'imposent pas l'hypothèse de rendements d'échelle constants, si bien qu'ils estiment le coefficient de la quantité de travail. Leur résultat est rappelé dans le tableau 2 et l'effet du capital humain est cette fois non-significatif. Pritchett (1996) estime également cette spécification avec les données de Barro \& Lee (1993) et de Nehru, Swanson \& Dubey (1995) et obtient des effets négatifs et parfois significatifs ${ }^{8}$. Ainsi, il existe à ce stade une contradiction manifeste entre les deux approches, par l'équation de convergence et par la fonction de production, les unes faisant apparaître l'effet positif du capital humain sur la croissance qui est attendu, les autres étant incapables de le mettre en évidence.

\section{Les biais d'endogénéité}

Afin de se concentrer sur la discussion des spécifications économiques, nous avons volontairement laissé de côté la discussion des méthodes économétriques utilisées pour estimer les différents modèles. Or ces méthodes ne sont légitimes que sous une hypothèse forte: que les variables explicatives du modèle, taux d'investissment, stocks de capital, croissance de la population ou encore produit par tête initial, soient indépendantes des caractéristiques non-observées qui distinguent les différents pays. L'existence de telles caractéristiques, et leur effet sur la

7 Leurs résultats sont robustes à l'utilisation des données de Barro \& Lee (1993).

8 La différence entre les estimations de Benhabib \& Spiegel (1994) et Pritchett (1996) tient principalement à l'introduction explicite de la quantité de travail par les premiers et à l'expression du stock de capital humain en valeur par le second (en utilisant le taux de rendement de l'éducation traité comme un prix). 
croissance ou le niveau du produit, sont pourtant attestées par l'introduction d'indicatrices régionales dans les estimations : les indicatrices pour l'Afrique sub-saharienne et pour l'Amérique latine sont presque toujours significatives. Peut-on raisonnablement supposer que ces caractéristiques sont sans effet sur les variables explicatives?

Pour clarifier la discussion, il convient de distinguer, dans le résidu non-observable, des caractéristiques constantes dans le temps et des événements dont la nature et l'ampleur peuvent varier d'une période à l'autre. Formellement, on se souvient que la composante du progrès technique dans la fonction de production peut s'écrire $\log A_{o}=a+\varepsilon$ où $\varepsilon$ est un résidu d'espérance nulle et $a$ la valeur moyenne de $\log A_{o}$. Supposons que $\varepsilon$ se décompose lui-même en une composante individuelle constante dans le temps et un résidu variable, $\varepsilon_{i t}=u_{i}+\nu_{i t}$. Pour estimer les modèles (3.1), (3.4) ou (3.5) par les moindres carrés ordinaires, il faut admettre que

$$
E\left(\varepsilon_{i t} \mid x_{i t}\right)=0
$$

(où $x$ représente l'ensemble des variables explicatives) et en particulier que

$$
E\left(u_{i} \mid x_{i t}\right)=0
$$

Les estimations de Barro (1991), Mankiw, Romer \& Weil (1992) et Islam (1995) que nous avons présentées ont recours à cette hypothèse contestable. Lorsque le modèle est estimé sous la forme (3.2) en revanche, le residu devient $\varepsilon_{i t}-\varepsilon_{i o}=\nu_{i t}-\nu_{i o}$ et les estimations sont robustes à l'hypothèse sur la corrélation entre l'effet fixe $u_{i}$ et les variables du modèle. Le problème de la corrélation avec la composante $\nu_{i t}$ reste cependant entier, mais nous discuterons cette deuxième difficulté plus loin. Le point le plus important est que les deux jeux d'estimations, celles résumées dans le tableau 1 et celles résumées dans le tableau 2 , qui conduisent à des conclusions inverses, correspondent à des spécifications dans lesquelles l'effet fixe $u_{i}$ est respectivement présent et absent. Il est donc tentant de conclure que les résultats qui font apparaître un rôle positif du capital humain sont des résultats biaisés par la présence de l'effet fixe, tandis que les autres seraient plus robustes ${ }^{9}$. Ce point fait l'objet de la contribution d'Islam (1995). Après avoir présenté le résultat que nous avons reporté dans le tableau 1, cet auteur exploite la dimension de panel de ses données en utilisant une observation par pays tous les cinq ans, au prix d'une légère réduction de la taille de son échantillon. Il estime alors le modèle (3.5) mais en spécifiant explicitement l'effet fixe $u_{i}$ de manière à obtenir des estimations non biaisées ${ }^{10}$. Ses estimations sont reportées dans le tableau 3 et elles

9 Au demeurant, la prise en compte d'un effet individuel permet de relâcher l'hypothèse d'un taux de croissance unique du progrès technique : en effet, la constante qui comprenait le terme $g t$ est maintenant individualisée.

10 Il utilise la méthode de Chamberlain (1982); d'autres méthodes, celle des moments généralisés et celle de Balestra \& Nerlove (1966) sont utilisées pas Dessus (1998) et donnent des résultats semblables. 
confirment que, lorsque les effets fixes sont pris en compte, le coefficient du capital humain n'est plus positif et peut être significativement négatif. Ce résultat démontre qu'il ne convient pas d'opposer les spécifications de convergence vers l'équilibre stationnaire aux spécifications de fonction de production, mais plutôt les estimations qui ne tiennent pas compte de l'effet fixe, qui donnent des résultats positifs mais sont potentiellement biaisées et les estimation qui corrigent ce biais et qui ne font pas apparaître d'effet positif, quelle que soit la spécification économique de référence. En d'autres termes, lorsque la présence d'effets fixes est prise en compte, le capital humain n'a plus d'effet positif sur la croissance, quel que soit le modèle de référence (convergence ou fonction de production).

Tableau 1 : Les coefficients du capital humain dans les spécifications de convergence

\begin{tabular}{ccc}
\hline Auteurs & Variable de capital humain & Estimation \\
\hline Barro (1991) & Taux de scolarisation primaire 1960 & 0.0181 \\
& & (0.0060) \\
& Taux de scolarisation secondaire 1960 & 0.0225 \\
& & $(0.0090)$ \\
Mankiw, Romer & Taux de scolarisation secondaire & 0.233 \\
\& Weil (1992) & (moyenne 1960-1985) & (0.060) \\
Islam (1995) & Stock de capital humain en 1985 & 0.1823 \\
& (données de Barro \& Lee, 1993) & (0.0895) \\
\hline
\end{tabular}

Source pour Mankiw, Romer \& Weil (1992) : tableau V; pour Barro (1991) : tableau IV; pour Islam (1995) : tableau V. Ecarts-types entre parenthèses.

Cette conclusion doit être nuancée par plusieurs remarques. La première est que l'estimation du modèle (3.5) en panel revient à supposer qu'à chaque date, tous les cinq ans sur la période 1960 à 1985, les économies sont proches de leur équilibre stationnaire : une proximité pour le moins fuyante. Il faut donc admettre que la cible de l'équilibre stationnaire se modifie au cours du temps, ce qui revient en principe à supposer des chocs exogènes sur les taux d'investissement ou leurs déterminants.

La deuxième remarque nous semble plus sérieuse. Malgré le soin apporté par les divers auteurs à la constitution des données de stock de capital humain, la difficulté de l'exercice rend inévitable l'introduction d'erreurs de mesure importantes, d'autant que les données originales, les taux de scolarisation enregistrés par l'UNESCO, sont elles-mêmes 
Tableau 2: Les coefficients du capital humain dans les spécifications de fonctions de production

\begin{tabular}{ccc}
\hline Auteurs & Variable de capital humain & Estimation \\
\hline Benhabib & Taux de croissance du stock & 0.063 \\
\& Spiegel (1994) & (données de Kyriacou, 1991) & (0.079) \\
Pritchett (1996) & Taux de croissance du stock & -0.049 \\
& (données de Barro \& Lee, 1993) & (0.046) \\
& Taux de croissance du stock & -0.104 \\
& (données de Nehru, Swanson \& Dubey, 1995) & (0.050) \\
\hline
\end{tabular}

Source pour Benhabib \& Spiegel (1994) : tableau 1; pour Pritchett (1996) : tableau 1.

Ecarts-types entre parenthèses.

suspectes (Behrman \& Rosenzwieg, 1994) ${ }^{11}$. Bosca, de la Fuente \& Domenech (1996) analysent en détail les incohérences qui existent par exemple entre les données de Barro \& Lee (1993) et celles de Nehru, Swanson \& Dubey (1995) y compris dans le sous-ensemble des pays de l'OCDE, pour lesquels les données statistiques d'origine devraient être les meilleures. Ils relèvent en outre un certain nombre d'invraisemblances dans l'évolution des stocks construits par Barro \& Lee (1993). Il est donc clair que les estimations présentent des biais d'erreur de mesure. Lorsqu'une variable est mesurée avec erreur, on sait que le coefficient estimé est biaisé vers zéro, simplement parce que sa corrélation avec la variable expliquée devient moins nette. Cela pourrait expliquer qu'un effet significatif estimé sur les taux de scolarisation (mesurés avec peu d'erreur) devienne non-significatif lorsqu'on passe aux données de stock (mesurées avec d'avantage d'erreur). En outre, le fait de passer en différences premières (comme lorsqu'on passe du modèle (3.1) au modèle (3.2)) a pour effet d'aggraver l'erreur de mesure, car la différence des stocks cumule les erreurs sur chacun des deux stocks de départ. Dans ces conditions, on peut craindre que le coefficient du capital humain n'ait plus d'effet significativement positif lorsqu'on tient compte des effets fixes, non pas parce qu'on a supprimé un biais d'endogénéité mais parce qu'on a aggravé l'erreur de mesure. Cependant, cette interprétation explique mal les coefficients significativement négatifs obtenus dans certains cas (tableaux 2 et 3$)^{12}$. D'autre part, lorsque plusieurs variables du modèle sont mesurées avec erreur

11 On notera par conséquent que les problèmes d'erreur de mesure ne concernent pas seulement les contributions qui utilisent les données de stock, même s'ils y sont probablement plus sévères.

12 En théorie, l'erreur de mesure peut produire des estimations négatives de coefficients théoriques positifs, mais pour des rapports bruit-signal extrêment forts. 
- et c'est au moins le cas du capital physique ${ }^{13}$-, les sens des biais sur les différents coefficients sont a priori indéterminés.

Tableau 3: Les coefficients du capital humain dans l'estimation en panel de Islam (1995)

\begin{tabular}{ccc}
\hline Echantillon de pays & Variable de capital humain & Estimation \\
\hline Non-pétrioliers & Stock de capital humain & -0.0712 \\
& (données de Barro \& Lee, 1993) & (0.0323) \\
Intermédiaires & Stock de capital humain & -0.0027 \\
& (données de Barro \& Lee, 1993) & (0.0471) \\
OCDE & Stock de capital humain & -0.0208 \\
& (données de Barro \& Lee, 1993) & (0.0449) \\
\hline
\end{tabular}

Source : tableau V. Ecarts-types entre parenthèses.

La troisième remarque concerne la corrélation des variables explicatives avec la composante $\nu_{i t}$ du résidu, laquelle n'est pas traitée par la prise en compte des effets fixes. Cette corrélation peut avoir des raisons économiques (un choc sur la productivité devrait affecter l'accumulation des facteurs) mais elle résulte également de l'erreur de mesure (l'erreur de mesure est un aléa corrélé avec la variable observée - laquelle est mesurée avec erreur - et qui passe dans le résidu ${ }^{14}$ ). Le traitement de cette forme d'endogénéité est donc aussi à la fois un traitement de l'erreur de mesure. Cette question est analysée par Benhabib \& Spiegel (1994) qui soutiennent que sous certaines hypothèses raisonnables, le biais sur la variable de capital humain qui pourrait être introduit par son endogénéité serait positif, si bien que leurs conclusions resteraient qualitativement inchangées (la croissance du capital humain n'affecte pas la croissance du produit). Une autre approche consiste à utiliser des méthodes instrumentales : il faut pour celà disposer de variables corrélées avec la variable endogène mais qui n'ont aucun effet direct sur le produit $y$. Ainsi, Pritchett (1996) utilise les données de Barro \& Lee (1993) pour instrumenter les stocks d'éducation de Nehru, Swanson \& Dubey (1995) puis il utilise ces derniers pour instrumenter les premiers. Cette méthode ne vaut que si les erreurs de mesure dans l'une et l'autre base sont non-corrélées entre elles. Il utilise par ailleurs, pour chaque pays, le stock d'éducation dans un pays semblable, souvent frontalier. Dans les deux cas, les résultats sont inchangés. En estimant le modèle (3.5) en panel, Dessus (1998) utilise

13 Pritchett (1996) souligne d'ailleurs que le stock de capital physique est fort mal mesuré, ce qui ne l'empêche pas d'avoir un coefficient significativement positif dans toutes les estimations. Pourquoi en irait-il différemment pour le capital humain si son effet était réellement positif ?

14 Ce point est présenté en détail par Greene (1997) par exemple. 
pour chaque période les données des autres périodes et observe également que l'instrumentation modifie peu les estimations. Bien qu'il soit toujours possible de discuter les hypothèses d'exogénéité des instruments, on manque d'arguments pour soutenir qu'il persiste des biais d'endogénéité importants, une fois pris en compte le rôle des effets fixes.

Notons enfin que, dans un modèle comme celui estimé par Islam (1995), seule la constante est autorisée à varier entre les pays du panel. Les autres coefficients, notamment le taux de croissance du progrès technique ou les coefficients des variables explicatives, sont supposés communs à tous les pays. Sous certaines hypothèses sur le comportement des variables du modèle en série temporelle, le fait d'imposer ces contraintes si elles sont fausses peut entraîner un biais vers 1 du coeffcient de progrès technique et vers 0 des coefficients des variables explicatives (Lee, Pesaran \& Smith, 1998). Malheureusement, les auteurs qui utilisent des techniques de panel dynamique en généralisant l'hétérogénéité des coefficients et en traitant avec soin la structure temportelle des termes stochastiques ${ }^{15}$ concentrent leur attention sur la problématique de la convergence et ne cherchent pas à identifier l'effet de l'accumulation du capital humain sur la croissance. Il est donc difficile d'évaluer l'effet de ces méthodes sur les résultats particuliers que nous discutons ici. Nous analyserons dans la section suivante la prise en compte de l'hétérogénéité des effets du capital humain, mais dans une perspective beaucoup moins économétrique.

À ce point, la littérature empirique semble donc dans une impasse : lorsque des méthodes économétriques soignées sont mises en œuvre, lorsqu'aucune hypothèse sur le processus d'accumulation du capital humain n'est mobilisée et quelle que soit la modélisation retenue (convergence vers l'équilibre ou fonction de production), il semble que les données résistent à décrire une relation positive entre le revenu agrégé et le capital humain.

\section{Les conditions de rentabilité du capital humain}

Certains développements de la littérature théorique sur la croissance endogène ont proposé de retirer le capital humain de la fonction de production (ou de relativiser le rôle qu'il y joue) et de l'introduire directement dans la modélisation de l'accroissement du progrès technique. C'est faire du capital humain non plus un facteur de production homogène au capital physique ou au travail mais une richesse à part, servant à la production de savoir et à l'innovation. C'est aussi donner

15 Voir par exemple Evans \& Karras (1996) Lee, Pesaran \& Smith (1997) et, pour une vision d'ensemble, Maddala (1999). 
une valeur économique différente à la fraction du capital humain qui est employée dans des activités d'amélioration de l'efficacité technique des économies et à celle qui est employée dans les activités directement productives. Ce type de modèles est essentiellement inspiré par l'article théorique de Romer (1990) : cet auteur suppose que le stock de capital humain employé dans des activités de recherche à un moment donné ne détermine pas le niveau du progrès technique mais son taux de croissance, soit

$$
\frac{\dot{A}}{A}=\phi H
$$

où $\phi$ est un paramètre de productivité. Cette relation est justifiée par l'hypothèse que les individus ont accès, pour produire un supplément de connaissances, à l'ensemble des connaissances disponibles, ce qui apparaît plus clairement si on écrit la relation sous la forme : $\dot{A}=\phi A H$. Cela implique que l'activité de recherche est d'autant plus productive que le stock de connaissances accumulées est important. Jones (1995) démontre cependant que cette structure est empiriquement incompatible avec des données en séries temporelles. Il suggère d'amender le modèle en écrivant $\dot{A}=\phi A H^{\psi}$, ce qui ne change pas la nature de la relation. Pour Aghion \& Howitt (1998), il convient donc d'opposer les modèles dans lesquels le taux de croissance de l'économie est déterminé par le taux de croissance du capital humain et ceux dans lesquels il est déterminé par son niveau.

Cette opposition est-elle confirmée empiriquement et la deuxième classe de modèles donne-t-elle de meilleurs résultats? Il nous semble que non et que le simple fait de substituer le niveau de capital humain à son taux de croissance ne produit aucun résultat utile. C'est l'expérience à laquelle se livrent Benhabib \& Spiegel (1994) dans une première étape. Ils estiment la relation suivante, proche de (3.2), à la variable de capital humain (et à l'hypothèse de rendements constants) près,

$\log Y_{i t}-\log Y_{i o}=\alpha\left(\log K_{i t}-\log K_{i o}\right)+\beta\left(\log L_{i t}-\log L_{i o}\right)+\gamma \overline{\log H}+\varepsilon_{i t}-\varepsilon_{i o}$

où $\overline{\log H}$ est une moyenne sur la période des logarithmes des stocks de capital humain, supposée déterminer le taux de croissance du progrès technique. Or les auteurs obtiennent un coefficient de $(-0.079)$ avec un écart-type de (0.060), ce qui permet difficilement de valider le modèle. Ils ajoutent ensuite le niveau de produit initial, $\log Y_{i o}$, en variable explicative afin de tenir compte d'un éventuel phénomène de convergence. Cette fois, l'estimateur de $\gamma$ devient positif et significatif. Mais cette deuxième relation est totalement ad hoc et elle s'apparente étrangement à l'équation (3.5) si on exprime cette dernière sous la forme

$\log \widetilde{y}_{i t}-\log \widetilde{y}_{i o}=\alpha^{\prime \prime} \log s_{k i}+\beta^{\prime \prime \prime} \log h_{i}^{*}-\gamma^{\prime} \log \left(n_{i t}+g+\delta\right)+(\theta-1) \log \widetilde{y}_{i o}+a+g t+\varepsilon_{i t}$ 
Or on sait que ce modèle, lorsqu'il est estimé sans tenir compte des effets fixes, produit un coefficient significatif pour le capital humain. On peut donc penser que le deuxième résultat de Benhabib \& Spiegel (1994) ne fait que reproduire le résultat d'Islam (1995) reporté dans notre tableau $1^{16}$. Bien sûr, les deux spécifications diffèrent en certains points (le taux d'investissement du capital physique est remplacé par son taux de croissance et $\log (n+g+\delta)$ est supprimé dans la version de Benhabib \& Spiegel); mais il n'est pas exclu que les deux modèles aient suffisamment de structure en commun pour qu'il soit difficile de les distinguer empiriquement. De façon générale, le modèle (3.5) fait figurer le capital humain en niveau dans l'explication du taux de croissance, alors même qu'il part d'une spécification classique de la fonction de production: son signe distinctif est la présence du produit retardé. Il faut donc être très attentif aux spécifications que l'on utilise si l'on veut opposer l'effet du capital humain en niveau et en taux de croissance. Et rien ne permet de conclure en faveur de la spécification en niveau.

Il nous semble que les quelques résultats empiriques qui permettent de réhabiliter le rôle de capital humain dans la croissance s'appuient sur une approche toute différente, même si elle s'accompagne parfois d'une spécification du capital humain en niveau. La plus grande partie de la littérature, en effet, néglige la possible hétérogénéité de l'effet du capital humain entre les pays ou à différentes périodes. Divers auteurs ont pourtant souligné que certaines circonstances sont favorables à la rentabilité du capital humain et d'autres moins (Schultz, 1975, Rosenzweig, 1995, Foster \& Rosenzweig, 1996). Dessus (1998) estime un modèle de la forme (3.5), mais en estimant un paramètre sur la variable de capital humain différent pour chaque pays : l'hypothèse d'homogénéité de l'effet du capital humain entre les pays est alors très largement rejetée. Il reste à analyser les sources des différences entre les pays. ${ }^{17}$

Dans une dernière étape de leur contribution, Benhabib \& Spiegel (1994) reprennent un modèle introduit par Nelson \& Phelps (1966) qui suggèrent que le capital humain sert principalement à importer et adopter les technologies nouvelles et proposent de spécifier le taux de croissance du progrès technique comme

$$
\frac{\dot{A}}{A}=m(H) \cdot \frac{A_{\max }-A}{A}
$$

où $A_{\max }$ mesure le niveau technique du pays le plus avancé et $m($.$) est$ une fonction. Dans ce modèle, la croissance d'un pays est d'autant

16 Et dont nous avons vu qu'il n'était pas robuste à la prise en compte des effets fixes.

17 La littérature économétrique récente utilise des méthodes de panel dynamique qui permettent d'estimer des modèles dont les paramètres sont hétérogènes et aléatoires entre les pays (voir Maddala, 1999). Nous nous intéressons ici à la littérature qui cherche à expliquer cette hétérogénéité. 
plus forte qu'il a un retard technologique important à rattraper, mais uniquement à condition qu'il dispose d'assez de capital humain pour réussir ce rattrapage. La nouveauté ne réside pas ici dans l'introduction du capital humain en niveau, mais bien plutôt dans son interaction avec le retard technologique. Benhabib \& Spiegel utilisent une forme différente de ce modèle, car ils distinguent l'innovation générée par le pays lui même et le rattrapage. D'autre part, ils expriment le rattrapage directement en fonction des produits agrégés, soit, avec des formes fonctionnelles linéaires,

$$
\frac{\dot{A}}{A}=g \cdot H+m \cdot H \cdot \frac{Y_{\max }-Y}{Y}=(g-m) \cdot H+m \cdot H \cdot \frac{Y_{\max }}{Y}
$$

Dans cette spécification, $g . H$ décrit l'innovation, c'est-à-dire la production directe de connaissances. Les résultats sont cette fois assez spectaculaire et ils sont résumés dans le tableau $4^{18}$. Le modèle est d'abord estimé sur l'ensemble de l'échantillon: le terme de rattrapage, estimé par le coefficient de $m$ est positif est significatif. Le terme $(g-m)$ en revanche est estimé avec beaucoup d'imprécision, si bien qu'il est impossible de rejeter l'hypothèse que $g$ soit nul ou même négatif. L'échantillon est ensuite divisé entre pays pauvres, pays intermédiaires et pays riches. Comme on s'y attend, le terme de rattrapage est important pour les pays les plus pauvres. A l'inverse, $m$ n'a pas d'effet significatif pour les pays les plus riches tandis que l'estimation de $(g-m)$ est plus précise, ce qui permet d'inférer une valeur de $g$ significativement positive, mais seulement au seuil de $10 \%$. Ainsi, le capital humain a un effet sur la croissance mais pour certains pays seulement: il permet le rattrapage technologique pour les plus pauvres et il favorise l'innovation pour les plus riches. En précisant davantage les voies par lesquelles il agit et en tenant compte de l'hétérogénéité entre les pays à cet égard, il est donc possible de confirmer empiriquement, quoiqu'avec des nuances, l'hypothèse que le capital humain aurait un effet positif sur la croissance économique.

Dessus (1998) présente un ensemble d'autres mécanismes qui pourraient également expliquer l'hétérogénéité des effets de l'éducation et il les estime dans le cadre du modèle (3.5) avec effets fixes, en croisant la variable de capital humain avec divers indicateurs. Dans le prolongement de l'approche précédente, il fait ainsi l'hypothèse que l'ouverture commerciale faciliterait la capacité des économies à utiliser les technologies étrangères, partant à procéder au rattrapage. Plusieurs mesures de l'ouverture économique sont utilisées, la part des échanges extérieurs dans le PIB, le tarif douanier moyen, la fréquence des barrières non-tarifaires et l'écart entre le taux de change officiel

18 La spécification reprend la fonction de production écrite en différences premières, ce qui supprime le biais d'effet fixe. Dans les résultats reportés dans notre tableau 4, il n'y a pas de niveau de production retardé. 
Tableau 4: Les coefficients du capital humain croisé avec d'autres variables

\begin{tabular}{ccc}
\hline Auteurs & Variable de capital humain & Estimation \\
\hline Benhabib & $H$ & $-0.0136(0.0144)$ \\
\& Spiegel (1994) & $H .\left(Y_{\max } / Y\right)$ & $0.0011(0.0002)$ \\
idem, pays pauvres & $H$ & $-0.0736(0.0586)$ \\
idem, pays riches & $H .\left(Y_{\max } / Y\right)$ & $0.0012(0.0003)$ \\
& $H$ & $0.0439(0.0224)$ \\
Dessus (1998) & $H .($ ouverture commerciale) & $0.864(0.123)$ \\
& $H .($ droits de propriété) & $0.113(0.027)$ \\
\hline
\end{tabular}

Source pour Benhabib \& Spiegel (1994) : tableau 5; pour Dessus (1998) : tableaux 2.1. et 2.4. Ecarts-types entre parenthèses.

et le taux du marché parallèle. L'effet du capital humain sur la croissance est alors d'autant plus élevé que l'économie est ouverte au sens de l'une ou l'autre de ces mesures (à l'exception de la fréquence des barrières non-tarifaires). Un indice qui synthétise l'ensemble des mesures commerciales est ensuite utilisé et son effet est significativement positif (tableau 4).

Un second ensemble de résultats proposés par Dessus (1998) conditionne la rentabilité du capital humain au cadre institutionnel des pays : un indice des libertés civiques, un indice de démocratisation, et une mesure de la fréquence des changements de gouvernement ${ }^{19}$. Leur effet croisé avec le capital humain est significatif et de signe attendu pour les deux premières mesures et non-significatif pour la dernière. Lorsqu'un indicateur synthétique de l'existence d'institutions de protection des droits de propriété (Sachs \& Warner, 1997) est utilisé, son effet est également significatif (tableau 4). Ces résultats confirment que la rentabilité du capital humain n'est pas acquise en toute circonstance, mais qu'elle est d'autant plus grande que certaines conditions sont réunies. L'interprétation précise du mécanisme en jeu est ici délicate. Dessus (1998) suggère que le capital humain n'est pas utilisé de façon optimale dans les économies où se développent les activités de recherche de rente et de corruption. Or ces activités seraient plus fréquentes lorsque les institutions garantissent moins bien les droits de propriété, l'application des contrats et les libertés civiques. C'est

19 Je renvoie le lecteur au texte de Dessus (1998) pour une définition détaillée de ces indicateurs. Il est entendu que leur signification et la simple possibilité de mesurer les phénomènes en question sont discutables mais leur examen documenté dépasse les objectifs de cet article. 
évidemment une interprétation et elle ne s'appuie pas sur l'estimation d'un modèle structurel.

Quoi qu'il en soit, cet ensemble de résultats est précieux parce qu'il alimente empiriquement l'hypothèse introduite par Benhabib \& Spiegel (1994) selon laquelle certaines conditions doivent être réunies pour que le capital humain ait effectivement un effet sur la croissance économique.

\section{Conclusion}

La conviction, généralement partagée, que le capital humain constitue bien un investissement socialement productif, d'abord confirmée par un ensemble de travaux, est aujourd'hui confrontée à un résultat empirique inattendu: les estimations les plus soignées, celles qui écartent au mieux les biais éventuels, sont incapables de démontrer que l'éducation est un facteur productif au niveau agrégé. Nous avons montré que la clef du passage d'un ensemble de résultats à l'autre est la prise en compte des caractéristiques constantes dans le temps et non-observées des pays. Si les pays qui ont des caractéristiques qui les rendent plus productifs accumulent aussi davantage de capital humain, une corrélation artificielle - ou surestimée - peut être trouvée entre la production et le capital humain. Lorsque ces effets fixes sont pris en compte, la corrélation disparaît, ce qui implique qu'il n'y aurait aucune relation directe, technique, entre le capital humain et le produit agrégé. Nous avons vu que les erreurs de mesure pourraient en théorie être à l'origine de ce résultat mais cette explication reste très insuffisante. En tout état de cause, on se trouverait dans l'impossibilité de mettre en évidence la relation empirique que l'on attend. Cette conclusion est en outre peu sensible à la modélisation utilisée : que l'on fasse l'hypothèse, peut-être contestable, que les économies sont proches de leur équilibre stationnaire et en mouvement vers celui-ci, ou que l'on estime directement une fonction de production sans faire d'hypothèse sur l'état d'équilibre des économies, les estimations convergent.

Il est habituel, dans cette littérature, non seulement de spécifier une fonction de production à la structure très simple mais surtout de supposer les paramètres de cette fonction constants dans le temps et dans l'espace. La raison tient principalement aux données limitées dont on dispose : pour la croissance de long terme, une donnée par pays, soit en général une centaine d'observations, et, si l'on accepte de découper cette croissance en épisodes de cinq ans, cinq à six fois plus. Dans le premier cas, il est difficile de donner beaucoup de souplesse aux modèles et, même si l'on admet en théorie une hétérogénéité des paramètres, on ne peut estimer autre chose que des effets moyens, ce qui n'est légitime que si l'hétérogénéité n'est pas trop importante. Un argument implicite ou explicite est la diffusion des technologies dans 
l'espace, mais c'est précisément cette diffusion qui est en jeu à travers les tests, au moins indirectement. Dans le second cas, on est en mesure d'introduire une dose d'hétérogénéité et on s'aperçoit alors qu'elle est extrêmement forte et qu'elle pourrait bien expliquer la médiocrité des estimations qui n'en tiennent pas compte.

Il nous semble qu'on identifie ainsi une cause possible du mal, mais que le diagnostic est encore incomplet. L'hétérogénéité s'explique manisfestement par la sensibilité du rendement du capital humain aux conditions économiques dans lesquelles il est exploité. C'est en soi un point important car il s'oppose à l'idée que les rendements du capital humain sont peut-être variables, mais, en toute circonstance, forts, idée véhiculée en partie par la littérature microéconométrique sur les équations de salaire. Nous n'affirmons pas que c'est inexact, nous soutenons simplement que les économistes ne sont pas en mesure de l'illustrer empiriquement au niveau macroéconomique et qu'il est par conséquent utile de remettre en question cette notion. Les rares contributions que nous avons évoquées mettent en œuvre un ensemble disparate de variables pour caractériser les situations qui pourraient affecter le rendement du capital humain. Manifestement, il manque encore une théorie des mécanismes exacts qui donnent au capital humain une valeur productive.

\section{Bibliographie}

Aghion P. \& Howitt P., Endogeneous Growth Theory, MIT Press, Cambridge, 1998.

Balestra P. \& Nerlove M., "Pooling cross-sections and time series data in the estimation of a dynamic model: the demand for natural gas", Econometrica, 1966, vol. 34, 585-612.

Barro R., "Economic Growth in a Cross Section of Countries", Quarterly Journal of Economics, 1991, vol. 151, n 2, May, 407-443.

Barro R. \& Lee J.-W., "International Comparisons of Educational Attainment”, Journal of Monetary Economics, 1993, vol. 32, $\mathrm{n}^{\circ} 3$, 363-394.

Barro J.R. \& Sala-i-Martin, X., Economic Growth, McGraw-Hill, New York, 1995.

Behrman J.R. \& Rosenzweig M.R., "Caveat Emptor; Cross-country Data on Education and the Labor Force", Journal of Development Economics, 1994, vol. 44, 147-171.

Benhabib J. \& Spiegel M.M., "The Role of Human Capital in Economic Development : Evidence from Aggregate Cross-Country Data", Journal of Monetary Economics, 1994, vol. 34, 143-179.

Bosca J., de la Fuente A. \& Domenech R., "Human Capital and Growth : Theory Ahead of Measurement”, mimeo, 1996. 
Chamberlain G., "Multivariate regression models for panel data", Journal of Econometrics, 1982, vol. 32, 189-218.

Cohen D., "Tests of the convergence Hypothesis : Some Further Results", Journal of Economic Growth, 1996, vol. 1, 351-361.

Dessus S., Analyses empiriques des déterminants de la croissance à long terme, Thèse de doctorat en sciences économiques, Université de Paris I, 1998.

Evans P. \& Karras G., "Convergence Revisited", Journal of Monetary Econonmics, 1996, vol. 37, 249-265.

Foster A.D. \& Rosenzweig M.R., "Technical Change and Human Capital Returns and Investments : Evidence from the Green Revolution", American Economic Review, 1996, vol. 86, n 4, September, 931953.

Greene W.H., Econometric Analysis, Macmillan, New York, 1997.

Islam N., "Growth Empirics : A Panel Data Approach", Quarterly Journal of Economics, 1995, November, 1127-1170.

Jones C.I., "Time Series Tests of Endogeneous Growth Models", Quarterly Journal of Economics, 1995, vol. 110, n 2, May, 495-526.

Kyriacou G., "Level and Growth Effects of Human Capital, A CrossCountry Study of the converence Hypothesis", Mimeo, NYU, May, 1991.

Lau L., Bhalla S. \& Louat F., "Human and Physical Stock in developing countries : Construction of Data and Trends", mimeo, World Bank, Washington, D.C., 1991.

Lau L., Jamison D. \& Louat F., Education and Productivity in Developing Countries : an Aggregae Production Function approach, World Bank Working Paper Series 612, World Bank, Washington, D.C., 1991.

Lee K., Pesaran M.H. \& Smith R., "Growth and Convergence in a MultiCountry Empirical Stochastic solow Model”, Journal of Applied Econometrics, 1997, vol. 12, 357-392.

Lee K., Pesaran M.H. \& Smith R., "Growth Empirics : A Panel Data Approach - a Comment”, Quarterly Journal of Economics, 1998, vol. 113, February, 319-323.

Levine R. \& Renelt D., "A Sensivity Analysis of Cross-Country Growth Regressions", American Economic Review, 1992, vol. 82, $\mathrm{n}^{\circ} 4$, 942-963.

Maddala, G.S., "On the Use of Panel Data Methods with Cross-Country Data", Annales d'économie et de statistique, 1999, n 55-56, 429448.

Mankiw N.G., Romer D. \& Weil D.N., "A Contribution to the Empirics of Economic Growth”, Quarterly Journal of Economics, 1992, vol. 107, May, 407-437.

Nehru V., Swanson E. \& Dubey A., "A New Database on Human Capital Stocks in Developing and Industrial Countries : Sources, Metho- 
dology and Results”, Journal of Development Economics, 1995, vol. $46, \mathrm{n}^{\circ} 2,379-401$.

Nelson R. \& Phelps E., "Investements in Humans, Technological Diffusion and Economic Growth", American Economic Review, 1966, vol. 61, $\mathrm{n}^{\circ}$ 2, May, 69-75.

Pritchett L., Where Has All the Education Gone?, World Bank Working Paper Series 1581, World Bank, Washington, D.C., 1996.

Romer P., "Endogeneous Technical Change", Journal of Political Economy, 1990, vol. 98, n 2, S71-S102.

Rosenzweig M.R., "Why are there Returns to Schooling", American Economic Review, 1995, vol. 85, ${ }^{\circ}$ 2, May, 153-158.

Sachs J. \& Warner A., "Fundamental Sources of Long-Run Growth", American Economic Review, 1997, vol. 87, 184-188.

Sala-i-Martin X., "I Just Ran Two Million Regressions", American Economic Review, 1997, vol. 87, ${ }^{\circ} 2$, 178-183.

Schultz T.W., "The Value of the Ability to Deal with Desequilibria", Journal of Economic Literature, 1975, vol. 13, 827-846.

Solow R. M., "A Contribution to the Theory of Economic Growth", Quarterly Journal of Economics, 1956, vol. 70, 65-94.

Swan T.W., "Economic Growth and Capital Accumulation", Economic Record, 1956, vol. 32, November, 334-361.

Zellner A., Kmenta J. \& Dreze J., "Specification and estimation of CobbDouglas Production Function Models", Econometrica, 1966, vol. 34, $\mathrm{n}^{\circ}$ 4, October, 784-795.

\section{Résumé}

Depuis le début des années 1990, la littérature empirique sur le rôle du capital humain dans la croissance économique fourmille de résultats contradictoires. Cet article récapitule les éléments du débat en opposant les contributions selon deux axes, celui de la spécification économique et celui des méthodes économétriques. Le premier axe oppose les estimations qui s'appuient sur des modèles de convergence des économies, à proximité de leur équilibre stationnaire et celles, de nature plus comptable, qui utilisent directement des fonctions de production. Le deuxième axe oppose les méthodes qui tiennent compte de la possible endogénéité du capital humain et celles qui la négligent. Lorsque les méthodes économétriques les plus robustes sont utilisées, il devient impossible de faire apparaître une relation positive entre le capital humain et la croissance ou le niveau du produit agrégé, quelle que soit la spécification économique retenue. L'erreur de mesure sur les données de stock de capital humain ne semble pas capable d'expliquer complètement ce résultat contre-intuitif. De rares travaux suggè- 
rent que la rentabilité de l'investissement en capital humain est très sensible à l'environnement économique, ce qui expliquerait la faiblesse des estimations qui supposent ce rendement homogène entre les pays. Cela doit modifier sensiblement notre conception du rôle économique du capital humain.

\begin{abstract}
Since the early 90's, the empirical literature on human capital in economic growth is full of contradictory results. This paper surveys the main contributions with emphasis on two axis : economic specification and econometric methods. Along the first axis, models based on convergence towards stationary equilibrium are opposed to production function estimates. Whether endogeneity of human capital is treated or not forms the second axis. Whenever robust econometric methods are used, a positive relationship between human capital and economic growth or the aggregate product does not come up, whichever economic specification is used. Measurement error on human capital stock data does not seem to account for this intruiguing result. A few papers indicate that the return to human capital may be sensitive to the economic environment, which may explain why estimates assuming homogeneous return between countries are weak. This should alter our way of thinking about human capital in the economy.
\end{abstract}

\title{
Mots-clés
}

capital humain, croissance (human capital, growth).

Classification JEL : I2, O 1, O4. 